\title{
Modern Techniques of Radiation Therapy in the Treatment of Brain Tumors and Tumors of the Skull Base
}

\section{(ㄷ) (1) (우)}

\author{
Authors \\ Christian Diehl' ${ }^{1}$ Stephanie E. Combs ${ }^{1,2}$
}

Affiliations
1 Department of Radiation Oncology and Radiation
Therapy, Technical University of Munich (TUM),
München, Germany
2 Institute of Innovative Radiotherapy (iRT), Department of
Radiation Sciences (DRS), Helmholtz Zentrum München,
Oberschleißheim, Germany

Keywords

intensity-modulated radiation therapy, IMRT, stereotaxy, gliomas, brain metastases, skull base tumors

\section{Bibliography}

DOI https://doi.org/10.1055/s-0043-119528

Neurology International Open 2017; 1: E97-E107

(c) Georg Thieme Verlag KG Stuttgart · New York

ISSN 2511-1795

Correspondence

Univ.-Prof. Dr. med. Stephanie E. Combs

Klinik für Radioonkologie und Strahlentherapie

Klinikum rechts der Isar

\author{
Technische Universität München (TUM) \\ Ismaninger Straße 22 \\ 81675 München \\ Germany \\ Stephanie.combs@tum.de
}

\begin{abstract}
Today the choice of radiation oncology technique and treatment concept is highly individualized. Modern techniques enable the use of high local doses with significant sparing of normal tissue. This has significantly improved the therapeutic window. Molecular markers guide therapy decisions within the interdisciplinary context, for primary as well as secondary brain tumors. Skull base tumors benefit from highly precise radiation techniques; often, radiation therapy can be seen as a treatment alternative to surgery in this region.
\end{abstract}

\section{Introduction}

Modern radiation oncology is one of the mainstays of multimodal treatment of primary and secondary brain tumors, especially if located at the skull base. Indications for radiation therapy are diverse and primarily based on histology, presenting symptoms, non-resectability of the tumor or status post partial resection. Individual treatment decisions as well treatment consensus in a multi-disciplinary team during tumor board meetings are important in all cases.

In primary brain tumors, such as gliomas, radiation therapy alone or in combination with systemic therapy is an important component of multidisciplinary management. Having attracted increasing attention in recent years, molecular tumor characteristics now significantly contribute to the treatment concept. In patients with secondary brain tumors (brain metastases), radiation therapy is typically used alone or sequentially with a systemic therapy which treats the extracranial manifestations of the disease.

Advantages of advanced radiation therapy include precise dose application to clearly defined areas so that normal tissue is spared, resulting in a reduced rate of side effects. Here, the precise definition of the volume to be treated (target volume) is crucial. Especially in the region of the skull base, long-term tumor control can be critical for patient outcomes; thus, special expertise is essential. In addition, high standards should be applied to the technology used. New technological developments allowing to adapt the radiation field to irregularly shaped target volumes at sub-millimeter scale have let to significantly improved sparing of surrounding normal tissue. However, enhanced precision entails high demands on radiation planning, i. e., on defining the radiation field.Intracranial space-occupying lesion account for a significant proportion of the tumor entities treated by radiation therapists and treatment planning and selection of the proper treatment regimen can be particularly challenging. Here, a thorough understanding of the techniques and evidence available is a basic requirement. 


\section{Techniques used in Modern Radiation Oncology}

Driven by continuous technological advances, radiation therapy has improved significantly over the last decades: Today, advanced linear accelerators (see - Fig. 1) provide high technical precision for effective tumor treatment and optimized normal tissue sparing.

Radiation therapy is always planned based on 3-dimensional dose calculations. First, head masks are formed to the individual patient, enabling daily repositioning for the various radiation treatment session. While the patient's head is immobilized using the

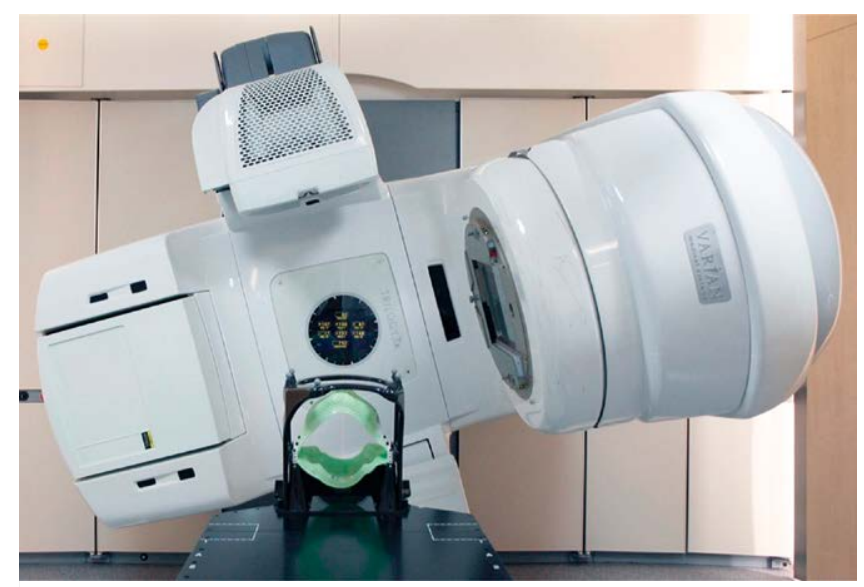

- Fig. 1 Modern linear accelerator with assembly for mask and stereotactic head frame.

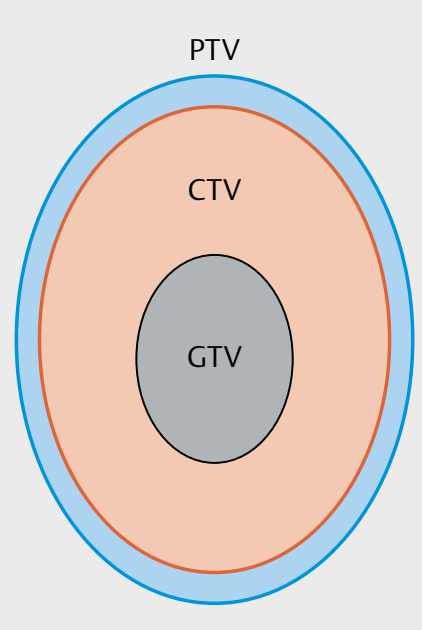

GTV Gross Tumor Volume "visible" tumor part/resection cavity

CTV Clinical Target Volume GTV $+1-2 \mathrm{~cm}$ Microscopic tumor extension

PTV Planning Target Volume CTV $+2-5 \mathrm{~mm}$ Movement and position change

- Fig. 2 Definition of target volumes. mask, a computed tomography (CT) scan is performed to determine the patient's position in the mask and to provide the data required for dose calculations. For the precise determination of the treatment volume, up-to-date imaging studies with and without contrast are required. Magnetic resonance imaging (MRI) should always be included in the work-up, unless contraindicated. Especially in the region of the skull base or for radiosurgery, MRI scanning should be performed as thin-sliced imaging (1-3 mm slice thickness); in addition, contrast-enhanced T1-weighted sequences and, especially for gliomas, T2-FLAIR sequences are relevant for planning. Next, a three-dimensional coordinate system is projected onto the patient, allowing to exactly allocate coordinates ( $x, y$, $z$ ) to every point of the patient. Then, the target volume is determined and structures at risk, such as brain stem, optic chiasm and optic nerve, are marked in each slice of the three-dimensional dataset. Depending on the indication, PET/CT or PET/MRI scans are frequently performed to define target volumes: for gliomas and brain metastases, amino acid PET, e. g., ${ }^{18}$ F-fluoro-deoxy-ethyl-tyrosine ( $\left.{ }^{18} \mathrm{~F}-\mathrm{FET}\right) \mathrm{PET}$; for meningiomas, somatostatin receptor PET (68Ga-DOTATOC PET). These radioactive tracers are taken up by tumor cells in a highly specific manner, showing as areas of particularly high activity in vital tumor tissue. This mechanism also allows to differentiate between genuine recurrences and treatment-associated changes [1,2]. The actual benefit of PET imaging for primary therapy planning and in the recurrence situation of intracranial tumors is currently being evaluated in studies.

Gross tumor volume (GTV) refers to the macroscopically identifiable tumor part or to the contrast-enhancing tumor areas. The suspected microscopic tumor infiltration zone is covered by the clinical target volume (CTV). In higher-grade gliomas, for example, a CTV of approximately $2 \mathrm{~cm}$ is included, while with benign space-occupying lesions, such as meningioma and acoustic neuroma, a CTV of only $1-2 \mathrm{~mm}$ is required. To allow for minor positioning inaccuracies, the CTV is expanded to form the planning target volume (PTV) which represents the actual target volume (see $>$ Fig. 2)

\section{Stereotactic Radiation Therapy/ Radiosurgery}

Precision of radiation therapy has improved over time: Especially the development of stereotactic radiation therapy by the neurosurgeon Lars Leksell contributed significantly to this progress:

It allowed point-precise application of high radiation doses with steep dose gradients at the margins of the radiation field. In this way, it is possible to apply very high local radiation doses as a single-session convergent beam irradiation (stereotactic radiosurgery). This approach is typically used to treat brain metastases, but also small acoustic neuromas or meningiomas [3-6]. Since with larger volumes the risk of side effects increases with single-session irradiation, fractionated concepts offering the same stereotaxic precision have also been developed [4, 5, 7-16]. With this approach, the total dose is spread over several fractions, allowing to use the radiation biological effect to reduce side effects. This method is referred to as fractionated stereotactic radiation therapy (FSRT). These techniques can be performed with linear accelerators specially designed for stereotaxy or with a Gamma Knife ${ }^{\circledR}$ or CyberKnife ${ }^{\circledR}$. Treatment outcomes are not influenced by the choice 
of radiation therapy equipment [17]. Today, invasive fixations which were required in the early days of radiosurgery are only used in individual cases. Today, precise head masks and exact imaging at the radiation therapy equipment are used to check and, if necessary, correct the daily position of the patient. Overall, special expertise of the radiation oncologist in these high-precision technologies is required to ensure adequate quality and safety.

\section{Intensity-Modulated Radiation Therapy (IMRT)}

Especially for target volumes with complex shapes, IMRT has brought major improvements: While achieving comparable precision, this technology allows to modulate individual radiation fields, i. e., there are subfields with higher or lower doses $[9,18,19]$. With this approach, the dose around high-risk organs can be planed. The capability to precisely treat or spare specific areas can also be used to apply higher doses of subvolumes. For example, it is possible to selectively target areas where biological imaging (e. g., PET/CT or $\mathrm{PET} / \mathrm{MRI}$ ) shows high tumor activity and treat them with higher doses. Various techniques, such as volumetric arc therapy (VMAT) or helical IMRT (Tomotherapie ${ }^{\circledR}$ ), are available for IMRT. These offer major advantages, especially if the target volume is very large, e. g., in case of entire neuroaxis radiation or simultaneous radiation of several target points. Especially at the skull base, helical IMRT (Tomotherapie ${ }^{\circledR}$ ) often ensures improved coverage of the target volume. This system's integration with an MV CT is crucial for positioning control, especially when metal implants were placed (in the area of the skull base or spine) during an earlier surgical procedure. The medical physicist calculates an individual radiation plan for the corresponding techniques to achieve optimum dose coverage of the target area (see $>$ Fig. $\mathbf{3}$ )

\section{Radiation Therapy with Protons and Heavy ions}

The physical characteristics of charged particles, for example protons or heavy ions (e. g., ${ }^{12} \mathrm{C}$ ions), used in radiation therapy differ from those of the photons used in conventional radiation therapy [20]. Immediately after penetrating into matter, photons release most of their energy. Particle radiation therapy shows an inverted depth-dose profile: When penetrating the body, the particles lose kinetic energy as the result of their interaction with matter. Initially, this energy loss is very small due to their velocity. However, it disproportionately increases with penetration depth and reaches its maximum at the end of the particle track when the particles have almost stopped. This phenomenon is referred to as "Bragg Peak", named after its discoverer, the British physicist Henry William Bragg. Consequently, the maximum of the corresponding energy dose is released at a defined point in the depth, while optimum tissue sparing is ensured for surrounding normal tissue (see - Fig. 4). This direct transfer of energy to tumor tissue, causing double-strand breaks and other damage, is referred to as linear energy transfer (LET). Since LET increases with increasing charge and mass, ions have a high LET in contrast to photons which have only an indirect effect. Relative biological effectiveness (RBE) is a measure of the damage done by a given type of radiation relative to

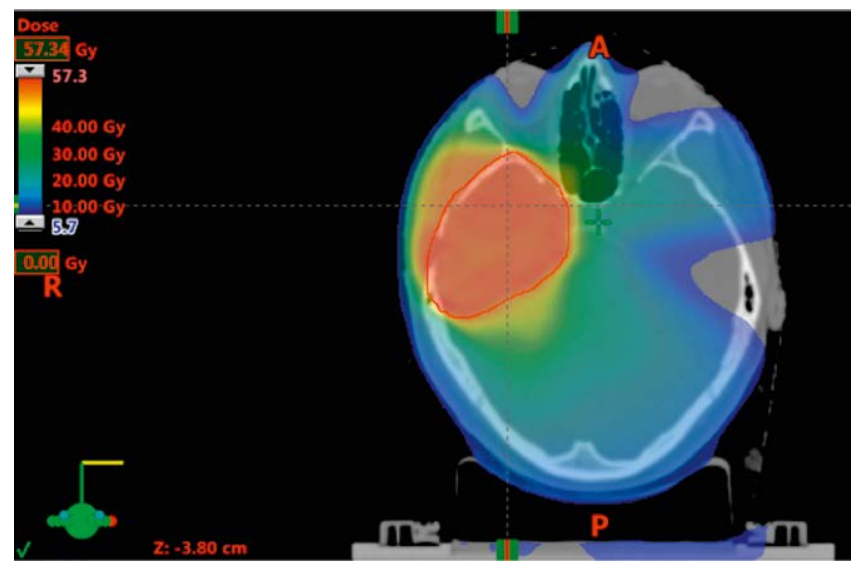

- Fig. 3 Color-coded dose distribution map of an IMRT plan for radiation therapy of a right temporal glioma. Red indicates highdose area.

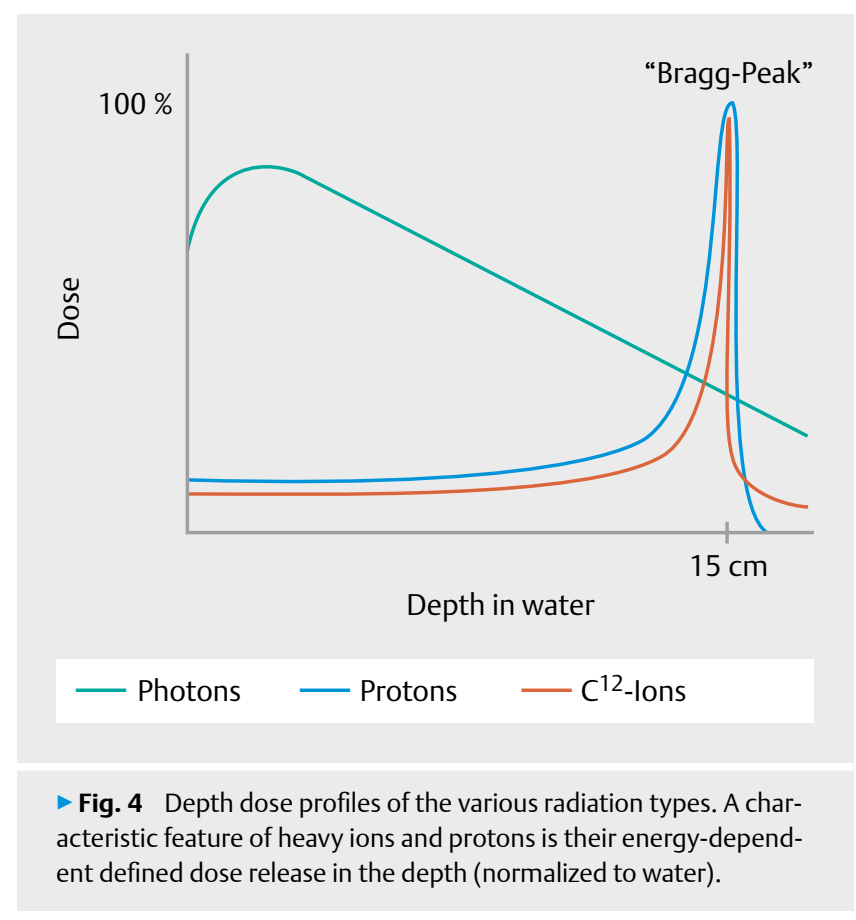

$250 \mathrm{keV} x$-rays. RBE of ${ }^{12} \mathrm{C}$ ions is higher than that of protons or photons and ranges between 2 and 5, depending on tumor type [2124]. By controlling velocity and consequently particle energy, the depth of the Bragg peak can be determined and the target volume focused accordingly. Especially for particle radiation therapy, the technical requirements are enormous and only available in a very limited number of centers. Currently, data on particle radiation therapy are sparse. Studies proving the superiority of particle therapy have not yet become available. Currently, the Cinderella study is comparing ${ }^{12} \mathrm{C}$ heavy-ion radiation therapy with conventional fractionated photon radiation therapy in patients with glioblastoma recurrence [13]. By contrast, the Cleopatra study investigates whether in the primary therapy of glioblastoma a local dose increase in the primary tumor region with ${ }^{12} \mathrm{C}$ heavy ions offers an advantage over a photon boost [25]. For both studies, interim anal- 
yses are currently prepared and first results are expected to be released soon.

The various techniques used in modern radiation oncology are selected based on tumor type, size and shape of the treatment area as well as the required radiation dose. Consequently, treatment is personalized in all cases and tailored to each individual patient's requirements and applied accordingly.

\section{Gliomas}

Gliomas originate from glial cells, the connective tissue in the central nervous system, and are classed according to the World Health Organization Classification of Tumors of the Central Nervous System based on their histological, immunohistochemical and molecular pathological characteristics. Essentially, it is differentiated between tumors with a better prognosis, such as low-grade gliomas (WHO grade I-II), and highly aggressive gliomas (WHO grade III-IV). According to the new 2016 WHO Classification of Tumors of the Central Nervous System [26], tumors are classified in a simplified way based on isocitrate dehydrogenase $1 / 2$ (IDH 1/2) mutations and the presence of an $1 p / 19 q$ codeletion (1p/19q CoDel):

An IDH mutation without $1 \mathrm{p} / 19 \mathrm{CoDel}$ is characteristic of astrocytoma. If in addition a codeletion of $1 p / 19 q$ is present, the tumor is an oligodendroglioma. Each of the two can be classed as anaplastic and consequently as WHO grade III. By contrast, histopathological diagnosis of mixed oligoastrocytoma which is based on light microscopic characteristics has lost their importance. Patients with an IDH wild-type have a very poor prognosis. In most grade III-IV cases, radiation therapy is indicated; however, malignancy grade, histopathological and molecular characteristics of the tumor as well as disease situation and age of patients also influence the management decision. Besides tumor resection and the available systemic therapies, radiation therapy plays a central role in the interdisciplinary treatment approach.Low-grade gliomas (WHO grade II) have considerably benefited in recent years from the identification of molecular markers, resulting in improved treatment success. Likewise, advances in radiation therapy have made treatment significantly more effective and gentler. Today's precision technologies allow an unprecedented degree of sparing of normal tissue. Higher radiation doses of up to 59.4 Gy in fractions of 1.8 Gy have not resulted in overall survival benefits compared with lower doses of e. g., 50.4 Gy, but are potentially associated with a profile of increased adverse events, including radiation necrosis. Therefore, standard treatment approaches commonly applied in clinical practice follow low-dose concepts of 45 to 54 Gy [27-29].

While radiation therapy was initially considered indicated in patients with tumor progression and after partial resection, the evidence available today shows that early postoperative radiation therapy after diagnosis prolongs progression-free survival. However, overall survival is not improved [30], a fact that needs to be individually discussed with patients. In addition, early initiation of radiation therapy is associated with improved seizure control after one year [30]. Furthermore, long-term results of an older study of the Radiation Therapy Oncology Group (RTOG) showed that highrisk patients with low-grade gliomas benefited from radiation therapy followed by 6 cycles of chemotherapy with PCV (procarbazine, CCNU, vincristine) [29]. High-risk patients included patients with incomplete neurosurgical tumor resection and/or aged older than 40 years. Identification of molecular markers can further aid selection of high-risk patients. A recent study has shown that in patients with $1 p / 19 q$ codeletion both progression-free survival and overall survival are significantly prolonged by adjuvant PCV chemotherapy after radiation therapy [31]. Additional prognostic markers include mutations of isocitrate dehydrogenase (IDH) and TERT promoter. For example, prognosis of WHO grade II astrocytomas without IDH mutation is comparably poor as that of WHO grade IV glioblastomas [32]. Whether chemotherapy with PCV can be replaced by temozolomide, especially because of the hematological toxicity profile, is being intensely discussed. In clinical practice, vincristine is frequently not administered. Currently, no randomized data are available to conclusively prove equal efficacy. However, survival data from recent studies have indicated that in patients with low-grade gliomas temozolomide chemotherapy may represent an alternative to PCV-based systemic therapy [33, 34]. In clinical practice, this approach has already been adopted for many cases [35]. However, a negative effect of PCV on neurocognition has not been demonstrated [36].

Based on our understanding of the timing and dose of radiation therapy for low-grade gliomas, molecular pathological risk profile-based individual radiation therapy concepts are conceivable. For example, oligodendrogliomas with IDH mutation and $1 \mathrm{p} / 19 \mathrm{q}$ codeletion could be treated with a lower total dose (e. g., 45$50.4 \mathrm{~Gy}$ ), while astrocytomas with unfavorable risk profile (IDH wildtype) could be treated with a higher total dose of up to $60 \mathrm{~Gy}$, similar to glioblastoma. Prospective randomized trials supporting the benefits of risk-adapted radiation therapy have not yet become available. With survival of potentially more than $5-10$ years, lowgrade gliomas with favorable molecular profile have a good prognosis; thus, long-term adverse effects, such as neurocognitive deficits, are of special interest. Numerous studies have investigated the potential effects of radiation therapy for tumors with low malignancy on cognition. It was found that the size of the radiation field correlates with deterioration of cognitive function [37]; this could be addressed by using new conformal techniques enabling minimization of radiation volumes. One study assessing cognition using a minimal mental test (MMSE) showed no decline in cognitive function; however, studies using more extensive test batteries are required before definite conclusions can be drawn [38]. Altogether, a potential negative effect of radiation therapy on neurocognition should be taken very seriously and needs to be discussed in detail with patients.

Another paradigm shift has occurred with regard to WHO grade III tumors: The traditional classification according to WHO grades has been modified, now integrating molecular markers and new insights into the biology of oligodendrogliomas. While some years ago discussions focused on the question whether radiation therapy could be replaced by chemotherapy [39], e. g., regimens based on PCV or temozolomide, today combined (sequential) chemoradiotherapy for some subtypes has become the center of attention. Long-term analysis of 2 large studies - one of EORTC, the other of RTOG - showed that the combination of radiation therapy and PCV significantly improved both progression-free survival and overall survival $[29,40]$. Definite study concepts now have to create insights into what molecular subgroups would particularly benefit 
Intensification of systemic therapy

- Alone or in combination with radiation therapy

- Depletion of repair mechanisms, e.g. MGMT

- Dose intensification of chemotherapy

\section{Use of molecular targets}

- Use of new molecular target substances

- Addition of molecular therapeutics to standard therapy

Immune system as a driver of treatment

- Immunomodulatory therapies

- Vaccinations

- Utilization of synergistic effects of radiation therapy and immune system

Individualized radiotherapy (iRT) based on glioma biology

- Stratification of dose concepts

- Molecular markers for personalization

- Radiation biological response as a stratification parameter

- Targeted use of combination therapies

- Utilization of growth and migration characteristics of gliomas

Radiation oncology treatment intensification

- Possibilities of high-precision radiation therapy

- Dose-increase strategies

- Use of new radiation qualities

- Dose painting and use of advanced imaging

- Fig. 5 Possible therapeutic strategies to optimize treatment of malignant gliomas.

from a combination therapy and which treatment sequence or type of systemic therapy offers the greatest benefits [41]. It should also be discussed from the perspective of radiation oncology which dose concepts will be adopted by modern radiation oncology: When subgroups of low-grade gliomas are redefined as high-risk gliomas, the radiation dose concept should be modified accordingly.

With an incidence of 5 cases per 100000 population, glioblastoma is the most common primary brain tumor, mostly affecting older people (peak incidence in the age group 60-70 years). Even after optimal resection and in the presence of favorable histological and molecular pathological features, this highly malignant invasive tumor has a very poor prognosis, with a median survival of $1-1.5$ years. Common initial signs and symptoms include sensomotor deficits, language disturbances, speech disturbances, and personality changes, as well as symptoms of increased intracranial pressure, including headache, nausea, vomiting and decreased alertness, depending on tumor location. Yet, new onset of seizures is also a common presentation leading to the diagnosis of glioblastoma. As a rule, management should always aim at complete neurosurgical removal of the tumor. Besides resection, adjuvant radiation therapy is a mainstay of glioblastoma treatment. For adjuvant concurrent chemoradiotherapy with temozolomide, a significant advantage over radiation therapy alone has been demonstrated. With this regimen, radiation therapy is administered up to a total dose of $60 \mathrm{~Gy}$, given in daily dose fractions of $2 \mathrm{~Gy}$. The significant impact of promotor-methylation of the repair enzyme O6-methylguanine-methyltransferase (MGMT) was already demonstrated in early studies (MGMT). Since then, several studies have used this criterion. In older patients (age > 70 years), especially among those in reduced general condition, treatment can be decided based on the methylation status of the MGMT promoter: In case of methylation, temozolomide treatment alone is a viable option, while in case of absence of promoter methylation an alternative approach based on radiation therapy as a hypofractionated concept with a total dose of $40.05 \mathrm{~Gy}$ administered in 15 individual doses can be used (in line with the NOA-08 study) [42]. However, more recent data show that these patients can also benefit from concurrent temozolomide treatment, with an acceptable risk profile [43]. Several management strategies can and have been pursued in patients with glioblastoma (see > Fig. 5). Large studies using molecular target substances in addition to or as an alternative to chemotherapy, have yielded negative results. Newer study concepts focus on glioblastoma biology and associated treatment resistance to develop individualized treatment pathways or leverage the immune system via checkpoint inhibitors: Tumor cells can escape the body's own immunological tumor defense by means of an overexpression of ligands which interact with the programmed cell death 1 (PD1) or the cytotoxic T-lymphocyte antigen-4 (CTLA-4) receptors on T-cells, thereby blocking their activation. The efficacy of so-called immune checkpoint inhibitors in glioma therapy, such as monoclonal anti-PD- 1 and anti-CTLA-4 antibodies, which have been used with considerable success in the treatment of malignant melanoma and non-small-cell lung cancer, among others, is currently being evaluated in randomized studies [44]. Since ionizing radiation has a significant effect on tumor cell immunogenicity [45], it is hoped that these new management approaches will yield positive results. Another avenue of research pursued is vaccination against glioma-specific antigens, such isocitrate dehydrogenase-I (IDH-1) which is frequently muted in higher-grade gliomas [46]. This approach is currently being explored in a phase I study (NOA$16 /$ NCT02454634) in combination with radiation therapy.

Despite all efforts in the primary situation, recurrences do occur, typically in the region of the primary tumor, i.e., within the radiation field. In these cases, the possibility of neurosurgical re-resection should be assessed. With the advent of technologies allowing high-precision radiation therapy, administering a second radiation therapy has become a treatment option, especially with intervals until recurrence of $>6$ months $[10,47]$. The optimal target volume for re-irradiation is currently being investigated in prospective studies: In patients with macroscopic residual tumor, the significance of amino acid PET for target volume definition is being investigated (GLIAA study), while the GlioCAVE study, led by the radiation oncology of the "Klinikum rechts der Isar" university hospital, is evaluating the role of re-irradiation after complete tumor resection.

Many times it has been speculated to what extent an intensified radiation therapy regimen could improve survival. However, hyperfractionated treatment concepts with 2 radiation sessions daily up to a total dose of $80 \mathrm{~Gy}$ could not detect any survival benefit [48]. 


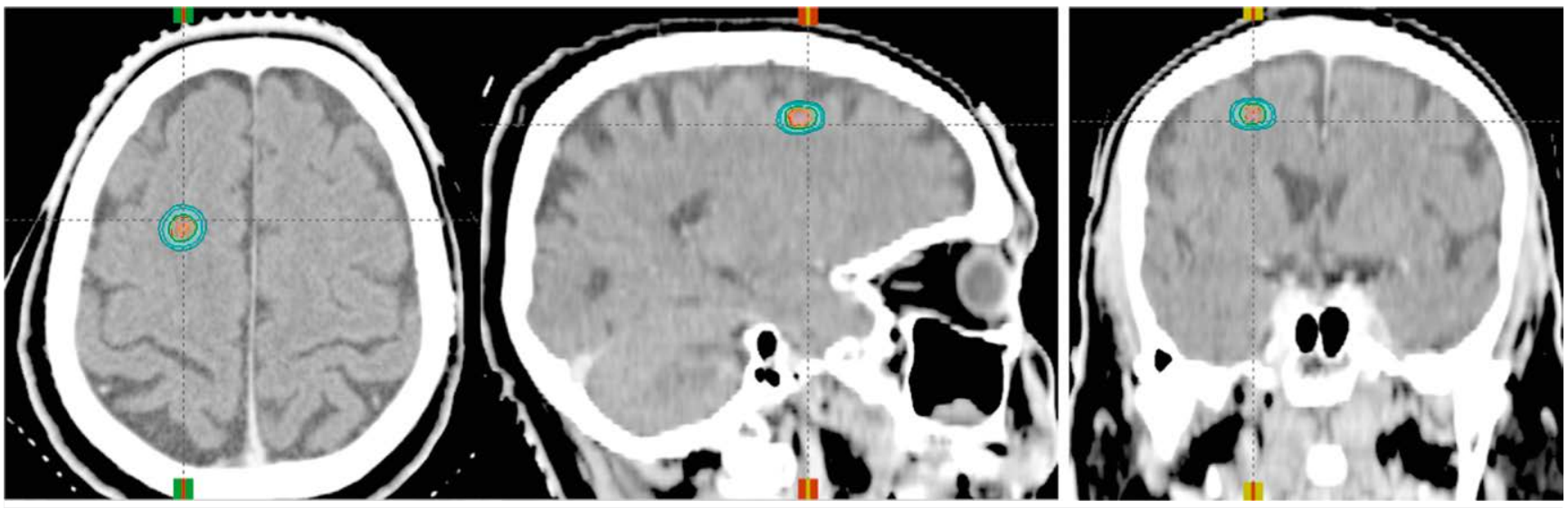

- Fig. 6 Radiosurgery of a patient with a brain metastasis from a non-small cell lung cancer (axial, sagittal and coronal views).

\section{Meningiomas}

Meningiomas are usually benign tumors, originating from the meningeal cells (cap cells) of the dura mater. The most common locations are the cerebral convexity and falx, followed by the posterior cranial fossa and skull base. Intraventricular meningiomas only account for a very small proportion of this tumor. Meningiomas are classified according to the WHO classification in grade I-III which has a decisive influence on the indication for radiation therapy. Altogether, WHO grade I meningiomas account for approximately $65-80 \%$ of all meningiomas and have a very favorable prognosis, provided complete microsurgical resection can be achieved. Depending on the location of the tumor, neurosurgical removal may be difficult or associated with a high morbidity risk. This is often the case with tumors of the skull base. Here, radiation therapy has developed into a highly effective and normal tissue-sparing treatment modality: either in addition as a consolidation therapy for residual tumor tissue, which is often deliberately not resected, or as an alternative to neurosurgical resection. Long-term results after high-precision radiation therapy show high local control of over $90 \%$ after 10 years [9]. Adequate imaging with MRI and computed tomography angiography (CTA) is an important prerequisite for radiation therapy of meningiomas as intraosseous parts of the tumor need to be revealed as well. Where ${ }^{68} \mathrm{Ga}$-Dotatoc PET is available, it can considerably contribute to optimizing the target volume for radiation therapy $[1,49,50]$. Treatment decisions, including preoperative decisions, should always be made by an interdisciplinary tumor board [51].

In high-risk meningiomas (WHO grade II and III), radiation therapy is central to achieving high local control. Small series have shown potential advantages of early postoperative radiation therapy for grade II meningiomas, too [52]. However, based on the evidence currently available, post-operative management of atypical meningioma can either rely on radiation therapy or follow-up checks at close intervals [53]. Preliminary data from ROTG 0539 indicate that postoperative radiation therapy in patients with WHO grade II meningiomas may prolong progression-free survival [54]. However, data from randomized trials have not yet become available. In patients with WHO grade III meningiomas, radiation therapy is clearly indicated because of the high recurrence rate [11].
Systemic therapies play a minor role in the management of meningioma.

\section{Vestibular Schwannoma (Acoustic Neuroma)}

Vestibular schwannomas are benign space-occupying lesions originating from the Schwann cells of the vestibular nerve. The term acoustic neuroma stems from the earlier assumption of a histological origin from the acoustic nerve. Vestibular schwannomas account for $10 \%$ of all intracranial space-occupying lesions. They can be located in the internal or external auditory meatus and have a growth rate of $1-3 \mathrm{~mm}$ per annum $[55,56]$, potentially growing to a considerable size ( $>4 \mathrm{~cm}$ ). For size-based grading, the Hannover classification can be used: While T1 tumors include intrameatal schwannomas, T3b tumors extend to the brain stem. In case of brain stem compression with or without displacement of the fourth ventricle, the tumor is classed as T4a or T4b [57]. In this situation or in patients with facial nerve paralysis, the first step should be to evaluate the possibility of neurosurgical decompression. Overall, surgical therapy of these larger tumors is clearly indicated; the other lesions can be treated with radiation therapy with comparable results [58]. Management of smaller, asymptomatic neuromas can initially be based on regular follow-up checks ("wait and scan") [59]. Since treatment decisions are also influenced by symptoms, age, comorbidities, and patient preferences, patients should receive detailed information about management with both radiation therapy and neurosurgery.

Fractionated stereotactic radiation therapy (FSRT) offers the most favorable treatment profile in radiation oncology. For FSRT, the likelihood of local control, including long-term control, is well in excess of $90 \%$, while the risk of adverse events, especially those involving cranial nerves, is well below $5 \%$. For smaller lesions, radiosurgery may be offered; however, great care should be taken when deciding about the radiation dose since with this method the radiation biological effect of fractionation cannot be exploited to reduce the risk of adverse events. Higher single doses ( >12 Gy) should be avoided as they significantly increase the risk of cranial nerve-related adverse events (facial nerve paralysis, trigeminal neuralgia, hearing loss) $[8,15]$. Provided careful radiation therapy plan- 

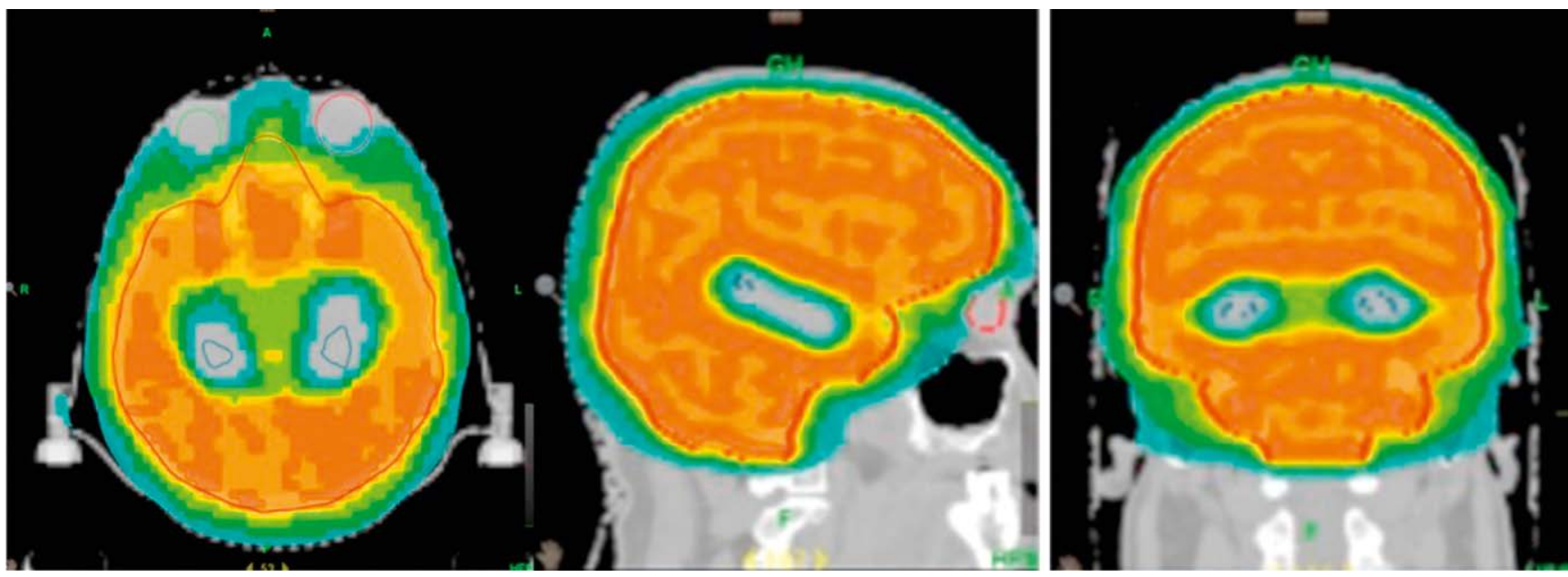

- Fig. 7 Modern radiation oncology techniques for whole-brain radiation therapy: Targeted hippocampal sparing to prevent neurocognitive deficits.

ning and dose calculation, both options can be safely used, primarily for smaller lesions [4].

\section{Other Indications for High-Precision Radiation Therapy}

Other intracranial space-occupying lesions which can be treated with radiation therapy are tumors of the sellar region, such as pituitary adenoma and craniopharyngioma. In the management of these two tumor types, high-precision radiation therapy can contribute to long-term tumor control (>90-95\%) with a very low risk of adverse events $[5,60,61]$.

\section{Secondary Brain Tumors (Metastases)}

Factors determining the treatment decision in patients with brain metastases include the number of lesions, the patient's general condition, previous treatments, and not least the underlying primary tumor. Altogether, metastases account for the majority of brain tumors treated with radiation therapy. Patients may have multiple metastases or one (singular or solitary) metastasis. A single brain metastasis is referred to as "solitary" if it is the only manifestation of tumor spread in the body. If additional extracerebral metastases are detected, the term "singulary" brain metastasis is used. However, multiple brain metastases are a common finding. The two types of cancer with the highest incidence rates in Germany and western industrialized countries - breast cancer in women and lung cancer in men - are the most common primary tumors underlying brain metastases; in young patients, malignant melanoma accounts for a significant proportion of brain metastases and is often associated with a high risk of bleeding [62].

Taking into account the size and number of metastatic lesions, radiation therapy offers several treatment options, including stereotactic concepts (e. g., radiosurgery) and whole-brain radiation therapy. Factors to be considered in the evaluation whether surgery and/or radiation therapy is indicated are number, size, and location of the metastases. Likewise, it is of importance whether neurological deficits or clinical signs of an acute increase in intracrani- al pressure are present. In most cases, the latter symptoms are caused by extensive perilesional edema and respond well to anti-edema therapy with dexamethasone. Depending on the severity of the symptoms, dexamethasone is administered either intravenously or orally, typically in doses of 8-24 mg per day with swift tapering to the maintenance dose. The most favorable management strategy for brain metastases is best decided by an interdisciplinary team during tumor board meetings. Besides the patient's staging status and general condition (comorbidities, Karnofsky performance status), current systemic therapies should be included in the decision making process. While some classical cytostatic drugs may significantly increase radiation neurotoxicity, especially during whole-brain radiation therapy, modern systemic therapies (antibodies, checkpoint modulators) may be used concomitantly, according to the evidence currently available. This approach is primarily used in patients not undergoing whole-brain but single-session high-dose radiation therapy (radiosurgery) [63-65]. Against the backdrop of rising life expectancy in cancer patients, increases in the numbers of patients requiring radiation therapy of the brain are to be expected. However, whenever novel agents, including immunomodulatory substances, are used, the indication for concurrent radiation therapy should always be critically assessed due to the potential of adverse effects in the radiation field. Furthermore, it is hoped that the radiation-induced exposure of antigens along with concomitant immunomodulatory reactivation of the body's defense system will result in an improved response of metastases located outside of the field (abscopal effect) [66].

Approximately $60 \%$ of patients with metastatic spread to the brain have more than 3 brain metastases at the time of diagnosis. The majority of these cases will be treated with whole-brain radiation therapy (WBRT). In patients with leptomeningeal spread of tumor cells, the radiation field is extended to include the $2^{\text {nd }}$ cervical vertebra (C2) ("German helmet" field). Dose prescription takes into account the underlying disease and the patient's life expectancy and may extend over a period of 2-4 weeks. In clinical practice, typically a dose of $10 \times 3$ Gy is applied. With this approach, median survival is 6 months, compared with 1-2 months for anti-edema therapy with dexamethasone alone (Best Supportive Care/BSC) 
[67]. For large metastatic lesions, IMRT offers the option of a local dose increase (boost), either as parallel or simultaneous integrated boost (SIB) or as a sequential boost subsequent to whole-brain radiation therapy. Especially concepts with low-dose fractions, e.g., a total dose of 40 Gy in 2 Gy fractions, are associated with a low risk of side effects, such as neurocognitive deficits. Particularly in patients with good prognosis and of young age, this should be taken into account when determining the treatment plan [68].

According to the evidence currently available, adjuvant WBRT after surgery or single-session radiation therapy (radiosurgery, see - Fig. 6) results in an improvement in locoregional progressions-free survical, but has no positive impact on overall survival [69]. Therefore, WBRT is not generally offered to patients with 1-3 (or even 4) brain metastases; instead, radiosurgical treatment options are selected. However, the treatment approach should be evaluated on an individual basis and the evidence currently available should be discussed with the patient. However, especially in cancers of radioresistant histologies, such as renal cell carcinoma or melanoma, high-dose focal single-fraction radiation offers a therapeutic advantage by achieving local tumor control [70]. The role of radiosurgery in patients with more than 3 (or 4 ) brain metastases remains the subject of controversy and should not be regarded as first-line therapy $[71,72]$.

After neurosurgical resection of a brain metastasis, the risk of recurrence is comparably high. Even though WBRT can improved local tumor control in these patients, it does not prolong survival, as discussed above. Here, precise local radiation of the resection cavity in the form of hypofractionated stereotactic radiation therapy (HFSRT) can improve local control [62, 73, 74].

For selected histologies, WBRT should not be avoided. This applies especially to small-cell lung cancer (SCLC), even if only few lesions are present in the brain. Here, prophylactic cranial irradiation (PCI) can significantly reduce the risk of developing brain metastases over the course of the disease and even provide a small survival benefit, if good tumor control is achieved [75]. Recently it has been shown that not only patients with localized tumors and regional lymph node involvement (limited disease), but also patients with distance metastasis (extensive disease) benefited from PCI [76].

Acute side effects of whole-brain radiation therapy include tiredness, confusion, nausea, and headache, frequently caused by perifocal edema. Impairment of cognitive performance can occur as a long-term side effect. It is a known fact that the hippocampus plays a decisive role in learning processes and memory formation, owing to the fact that the hippocampal formation contains neural stem cells in the dentate gyrus [77]. These can be damaged by radiation therapy [78]. Today, using advanced highly-conformal technologies, such as VMAT or helical IMRT (Tomotherapie ${ }^{\circledR}$ ), it is possible to spare the hippocampus (see $>$ Fig. 7) during whole-brain radiation therapy $[79,80]$. The potential of hippocampal avoidance (HA) to reduce the risk of impairment of cognitive function has already been demonstrated in studies. Currently, the NOA-14 study (HIPPORAD) investigates the effect of hippocampal avoidance during whole-brain radiation therapy of 4-10 metastatic lesions on cognitive performance. Overall, metastases very rarely occur in the hippocampus; thus, the increase in risk of metastases developing in the untreated hippocampal region is low with $0.2 \%$ [81].

\section{Conclusion and Outlook: Individualized} Radiotherapy (iRT)

Radiation therapy is an important therapeutic tool in the treatment of brain tumors and tumors of the skull base. With rising life expectancy, an increase in the incidence of brain tumors is to be expected. Even though surgery remains the mainstay of brain tumor treatment, radiation therapy is gaining in popularity, as it represents a valid, non-invasive and often normal tissue-sparing treatment alternative, especially for benign lesions such as meningioma and acoustic neuroma. In secondary brain tumors, too, high-precision radiation therapy is regarded as a method which is equivalent in effectiveness to resection in the treatment of singulary metastases. Advanced imaging with PET/MRI or PET/CT and thin-sliced MRI sequences has not only helped to optimize radiation therapy planning and further differentiate the treatment area, but also to detect metastases and recurrences early and treat them timely with radiation therapy. Technical innovations have optimized and increased the precision of radiation therapy, making it more effective with less side effects: Intraoperative radiation therapy enables the application of effective high radiation doses to the resection bed - the site where most recurrences occur - while the patient is still in the operating room. This could not be achieved with conventional percutaneous radiation therapy. Likewise, improved identification of navigated eloquent brain areas, such as the motor cortex und Broca area, helps the radiation therapist to develop a treatment plan that better spares this areas and prevents long-term side effect. Thus, the use of navigated transcranial magnetic stimulation (nTMS) for cortex mapping - already routinely applied in neurosurgery planning - is conceivable for radiation therapy planning.

Besides technical innovations, molecular pathological characteristics of brain tumors may have a decisive impact on radiation therapy concepts with regard to start of treatment, fractionation and radiation dose in the future. This would have a significant impact on interdisciplinary decision making and may - in combination with novel immunomodulating systemic therapies - allow us to offer every patient a personalized treatment strategy in the future, making individualized radiotherapy (iRT) a clinical reality. Numerous studies have been evaluating the potential of new immunogenic antibodies in the combination therapy of brain tumors, but reliable data have not become available as yet.

\section{Conflict of Interest}

No conflict of interest has been declared by the authors.

References

[1] Afshar-Oromieh A, Wolf MB, Kratochwil C et al. Comparison of (6)(8) Ga-DOTATOC-PET/CT and PET/MRI hybrid systems in patients with cranial meningioma: Initial results. Neuro Oncol 2015; 17: 312-319

[2] Rieken S, Habermehl D, Giesel FL et al. Analysis of FET-PET imaging for target volume definition in patients with gliomas treated with conformal radiotherapy. Radiother Oncol 2013; 109: 487-492 
[3] Hauswald H, Stenke A, Debus ] et al. Linear accelerator-based stereotactic radiosurgery in 140 brain metastases from malignant melanoma. BMC Cancer 2015; 15: 537

[4] Combs SE, Engelhard C, Kopp C et al. Long-term outcome after highly advanced single-dose or fractionated radiotherapy in patients with vestibular schwannomas - pooled results from 3 large German centers. Radiother Oncol 2015; 114: 378-383

[5] Harrabi SB, Adeberg S, Welzel T et al. Long term results after fractionated stereotactic radiotherapy (FSRT) in patients with craniopharyngioma: maximal tumor control with minimal side effects. Radiat Oncol 2014; 9: 203

[6] Combs SE, Schulz-Ertner D, Thilmann C et al. Treatment of cerebral metastases from breast cancer with stereotactic radiosurgery. Strahlenther Onkol 2004; 180: 590-596

[7] Wagner ], Welzel T, Habermehl D et al. Radiotherapy in patients with vestibular schwannoma and neurofibromatosis type 2: Clinical results and review of the literature. Tumori 2014; 100: 189-194

[8] Combs SE, Welzel T, Kessel K et al. Hearing preservation after radiotherapy for vestibular schwannomas is comparable to hearing deterioration in healthy adults and is accompanied by local tumor control and a highly preserved quality of life (QOL) as patients' self-reported outcome. Radiother Oncol 2013; 106: 175-180

[9] Combs SE, Adeberg S, Dittmar JO et al. Skull base meningiomas: Long-term results and patient self-reported outcome in 507 patients treated with fractionated stereotactic radiotherapy (FSRT) or intensity modulated radiotherapy (IMRT). Radiother Oncol 2013; 106: 186-191

[10] Combs SE, Edler L, Rausch R et al. Generation and validation of a prognostic score to predict outcome after re-irradiation of recurrent glioma. Acta Oncol 2013; 52: 147-152

[11] Adeberg S, Hartmann C, Welzel T et al. Long-term outcome after radiotherapy in patients with atypical and malignant meningiomas--clinical results in 85 patients treated in a single institution leading to optimized guidelines for early radiation therapy. Int J Radiat Oncol Biol Phys 2012; 83: 859-864

[12] Adeberg S, Welzel T, Rieken S et al. Prior surgical intervention and tumor size impact clinical outcome after precision radiotherapy for the treatment of optic nerve sheath meningiomas (ONSM). Radiat Oncol 2011; 6: 117

[13] Combs SE, Burkholder I, Edler L et al. Randomised phase I/II study to evaluate carbon ion radiotherapy versus fractionated stereotactic radiotherapy in patients with recurrent or progressive gliomas: The CINDERELLA trial. BMC Cancer 2010; 10: 533

[14] Combs SE, Hartmann C, Nikoghosyan A et al. Carbon ion radiation therapy for high-risk meningiomas. Radiother Oncol 2010; 95: 54-59

[15] Combs SE, Welzel T, Schulz-Ertner D et al. Differences in clinical results after LINAC-based single-dose radiosurgery versus fractionated stereotactic radiotherapy for patients with vestibular schwannomas. Int J Radiat Oncol Biol Phys 2010; 76: 193-200

[16] Combs SE, Steck I, Schulz-Ertner D et al. Long-term outcome of high-precision radiotherapy in patients with brain stem gliomas: Results from a difficult-to-treat patient population using fractionated stereotactic radiotherapy. Radiother Oncol 2009; 91: 60-66

[17] Andrews DW, Scott CB, Sperduto PW et al. Whole brain radiation therapy with or without stereotactic radiosurgery boost for patients with one to three brain metastases: Phase III results of the RTOG 9508 randomised trial. Lancet 2004; 363: 1665-1672

[18] Kosaki K, Ecker S, Habermehl D et al. Comparison of intensity modulated radiotherapy (IMRT) with intensity modulated particle therapy (IMPT) using fixed beams or an ion gantry for the treatment of patients with skull base meningiomas. Radiat Oncol 2012; 7: 44

[19] Combs SE, Behnisch W, Kulozik AE et al. Intensity Modulated Radiotherapy (IMRT) and Fractionated Stereotactic Radiotherapy (FSRT) for children with head-and-neck-rhabdomyosarcoma. BMC Cancer 2007; 7: 177
[20] Combs SE, Schulz-Ertner D, Herfarth KK et al. [Advances in radio-oncology. From precision radiotherapy with photons to ion therapy with protons and carbon ions]. Chirurg 2006; 77: 1126-1132

[21] HabermehI D, Ilicic K, Dehne $S$ et al. The relative biological effectiveness for carbon and oxygen ion beams using the raster-scanning technique in hepatocellular carcinoma cell lines. PLoS One 2014; 9: e113591

[22] Schlaich F, Brons S, Haberer T et al. Comparison of the effects of photon versus carbon ion irradiation when combined with chemotherapy in vitro. Radiat Oncol 2013; 8: 260

[23] El Shafie RA, Habermehl D, Rieken S et al. In vitro evaluation of photon and raster-scanned carbon ion radiotherapy in combination with gemcitabine in pancreatic cancer cell lines. J Radiat Res 2013; 54: (Suppl 1): i113-i119

[24] Combs SE, Bohl J, Elsasser T et al. Radiobiological evaluation and correlation with the local effect model (LEM) of carbon ion radiation therapy and temozolomide in glioblastoma cell lines. Int J Radiat Biol 2009; 85: 126-137

[25] Combs SE, Kieser M, Rieken S et al. Randomized phase II study evaluating a carbon ion boost applied after combined radiochemotherapy with temozolomide versus a proton boost after radiochemotherapy with temozolomide in patients with primary glioblastoma: the CLEOPATRA trial. BMC Cancer 2010; 10: 478

[26] Louis DN, Perry A, Reifenberger G et al. The 2016 World Health Organization Classification of Tumors of the Central Nervous System: A summary. Acta Neuropathol 2016; 131: 803-820

[27] Karim AB, Maat B, Hatlevoll R et al. A randomized trial on dose-response in radiation therapy of low-grade cerebral glioma: European Organization for Research and Treatment of Cancer (EORTC) Study 22844. Int J Radiat Oncol Biol Phys 1996; 36: 549-556

[28] Shaw E, Arusell R, Scheithauer B et al. Prospective randomized trial of low- versus high-dose radiation therapy in adults with supratentorial low-grade glioma: Initial report of a North Central Cancer Treatment Group/Radiation Therapy Oncology Group/Eastern Cooperative Oncology Group study. J Clin Oncol 2002; 20: 2267-2276

[29] van den Bent M], Brandes AA, Taphoorn MJ et al. Adjuvant procarbazine, lomustine, and vincristine chemotherapy in newly diagnosed anaplastic oligodendroglioma: Long-term follow-up of EORTC brain tumor group study 26951. J Clin Oncol 2013; 31: 344-350

[30] van den Bent M], Afra D, de Witte O et al Long-term efficacy of early versus delayed radiotherapy for low-grade astrocytoma and oligodendroglioma in adults: the EORTC 22845 randomised trial. Lancet 2005; 366: 985-990

[31] Buckner JC, Shaw EG, Pugh SL et al. Radiation plus Procarbazine, CCNU, and Vincristine in Low-Grade Glioma. N Engl J Med 2016; 374: 1344-1355

[32] Eckel-Passow JE, Lachance DH, Molinaro AM et al. Glioma Groups Based on 1p/19q, IDH, and TERT Promoter Mutations in Tumors. N Engl J Med 2015; 372: 2499-2508

[33] Fisher B], Hu C, Macdonald DR et al. Phase 2 study of temozolomide-based chemoradiation therapy for high-risk low-grade gliomas: Preliminary results of Radiation Therapy Oncology Group 0424. Int J Radiat Oncol Biol Phys 2015; 91: 497-504

[34] Haque W, Verma V, Butler EB et al. Patterns of care and outcomes of multi-agent versus single-agent chemotherapy as part of multimodal management of low grade glioma. J Neurooncol 2017; 133: 369-375

[35] Field KM, Rosenthal MA, Khasraw $M$ et al. Evolving management of low grade glioma: No consensus amongst treating clinicians. J Clin Neurosci 2016; 23: 81-87

[36] Prabhu RS, Won M, Shaw EG et al. Effect of the addition of chemotherapy to radiotherapy on cognitive function in patients with low-grade glioma: secondary analysis of RTOG 98-02. J Clin Oncol 2014; 32: 535-541 
[37] Swennen MH, Bromberg JE, Witkamp TD et al. Delayed radiation toxicity after focal or whole brain radiotherapy for low-grade glioma. J Neurooncol 2004; 66: 333-339

[38] Brown PD, Buckner JC, O'Fallon JR et al. Effects of radiotherapy on cognitive function in patients with low-grade glioma measured by the folstein mini-mental state examination. J Clin Oncol 2003; 21: 2519-2524

[39] Wick W, Hartmann C, Engel C et al. NOA-04 randomized phase III trial of sequential radiochemotherapy of anaplastic glioma with procarbazine, lomustine, and vincristine or temozolomide. J Clin Oncol 2009; 27: $5874-5880$

[40] Cairncross G, Wang M, Shaw E et al. Phase III trial of chemoradiotherapy for anaplastic oligodendroglioma: long-term results of RTOG 9402. J Clin Oncol 2013; 31: 337-343

[41] Wiestler B, Capper D, Hovestadt V et al. Assessing CpG island methylator phenotype, $1 p / 19 q$ codeletion, and MGMT promoter methylation from epigenome-wide data in the biomarker cohort of the NOA-04 trial. Neuro Oncol 2014; 16: 1630-1638

[42] Wick W, Platten M, Meisner C et al. Temozolomide chemotherapy alone versus radiotherapy alone for malignant astrocytoma in the elderly: the NOA-08 randomised, phase 3 trial. Lancet Oncol 2012; 13: 707-715

[43] Perry JR, Laperriere N, O'Callaghan C] et al. Short-course radiation plus temozolomide in elderly patients with glioblastoma. N Engl J Med 2017; 376: 1027-1037

[44] Preusser M, Lim M, Hafler DA et al. Prospects of immune checkpoint modulators in the treatment of glioblastoma. Nat Rev Neurol 2015; 11: 504-514

[45] Kachikwu EL, Iwamoto KS, Liao YP et al. Radiation enhances regulatory T cell representation. Int J Radiat Oncol Biol Phys 2011; 81: 1128-1135

[46] Capper D, Zentgraf H, Balss J et al. Monoclonal antibody specific for IDH1 R132H mutation. Acta Neuropathol 2009; 118: 599-601

[47] Combs SE, Thilmann C, Edler L et al. Efficacy of fractionated stereotactic reirradiation in recurrent gliomas: long-term results in 172 patients treated in a single institution. J Clin Oncol 2005; 23: 8863-8869

[48] Fulton DS, Urtasun RC, Scott-Brown I et al. Increasing radiation dose intensity using hyperfractionation in patients with malignant glioma. Final report of a prospective phase I-II dose response study. J Neurooncol 1992; 14: 63-72

[49] Combs SE, Welzel T, Habermehl D et al. Prospective evaluation of early treatment outcome in patients with meningiomas treated with particle therapy based on target volume definition with MRI and 68Ga-DOTATOC-PET. Acta Oncol 2013; 52: 514-520

[50] Afshar-Oromieh A, Giesel FL, Linhart HG et al. Detection of cranial meningiomas: Comparison of (6)(8)Ga-DOTATOC PET/CT and contrast-enhanced MRI. Eur J Nucl Med Mol Imaging 2012; 39 : 1409-1415

[51] Combs SE, Ganswindt U, Foote RL et al. State-of-the-art treatment alternatives for base of skull meningiomas: Complementing and controversial indications for neurosurgery, stereotactic and robotic based radiosurgery or modern fractionated radiation techniques. Radiat Oncol 2012; 7: 226

[52] Hug EB, Devries A, Thornton AF et al. Management of atypical and malignant meningiomas: role of high-dose, 3D-conformal radiation therapy. J Neurooncol 2000; 48: 151-160

[53] Rogers L, Barani I, Chamberlain M et al. Meningiomas: knowledge base, treatment outcomes, and uncertainties. A RANO review. J Neurosurg 2015; 122: 4-23

[54] Rogers PZ, MA Vogelbaum A et al. Intermediate-Risk Meningioma: Initial Outcomes from NRG. Oncology/RTOG-0539. Int J Radiat Oncol Biol Phys 2015; 93: S139-S140
[55] Bakkouri WE, Kania RE, Guichard JP et al. Conservative management of 386 cases of unilateral vestibular schwannoma: Tumor growth and consequences for treatment. J Neurosurg 2009; 110: 662-669

[56] Walsh RM, Bath AP, Bance ML et al. The natural history of untreated vestibular schwannomas. Is there a role for conservative management? Rev Laryngol Otol Rhinol (Bord) 2000; 121: 21-26

[57] Samii M, Matthies C. Management of 1000 vestibular schwannomas (Acoustic Neuromas): The facial nerve-preservation and restitution of function. Neurosurgery 1997; 40: 684-695

[58] Pollock BE, Driscoll CL, Foote RL et al. Patient outcomes after vestibular schwannoma management: A prospective comparison of microsurgical resection and stereotactic radiosurgery. Neurosurgery 2006; 59: 77-85 discussion 77-85

[59] Raut VV, Walsh RM, Bath AP et al. Conservative management of vestibular schwannomas - second review of a prospective longitudinal study. Clin Otolaryngol Allied Sci 2004; 29: 505-514

[60] Combs SE, Thilmann C, Huber PE et al. Achievement of long-term local control in patients with craniopharyngiomas using high precision stereotactic radiotherapy. Cancer 2007; 109: 2308-2314

[61] Rieken S, Habermehl D, Welzel T et al. Long term toxicity and prognostic factors of radiation therapy for secreting and non-secreting pituitary adenomas. Radiat Oncol 2013; 8: 18

[62] Mehta MP, Tsao MN, Whelan TJ et al. The American Society for Therapeutic Radiology and Oncology (ASTRO) evidence-based review of the role of radiosurgery for brain metastases. Int J Radiat Oncol Biol Phys 2005; 63: 37-46

[63] Seyedin SN, Schoenhals JE, Lee DA et al. Strategies for combining immunotherapy with radiation for anticancer therapy. Immunotherapy 2015; 7: 967-980

[64] Tang C, Wang $X$, Soh $\mathrm{H}$ et al. Combining radiation and immunotherapy: A new systemic therapy for solid tumors? Cancer Immunol Res 2014; 2: 831-838

[65] Patel KR, Lawson DH, Kudchadkar RR et al. Two heads better than one? Ipilimumab immunotherapy and radiation therapy for melanoma brain metastases. Neuro Oncol 2015; 17: 1312-1321

[66] Okwan-Duodu D, Pollack BP, Lawson D et al. Role of radiation therapy as immune activator in the era of modern immunotherapy for metastatic malignant melanoma. Am J Clin Oncol 2015; 38: 119-125

[67] Tsao MN, Rades D, Wirth A et al. Radiotherapeutic and surgical management for newly diagnosed brain metastasis(es): An American Society for Radiation Oncology evidence-based guideline. Pract Radiat Oncol 2012; 2: 210-225

[68] Graham PH, Bucci J, Browne L. Randomized comparison of whole brain radiotherapy, 20 Gy in four daily fractions versus 40 Gy in 20 twice-daily fractions, for brain metastases. Int J Radiat Oncol Biol Phys 2010; 77: 648-654

[69] Kocher M, Soffietti R, Abacioglu U et al. Adjuvant whole-brain radiotherapy versus observation after radiosurgery or surgical resection of one to three cerebral metastases: Results of the EORTC 22952-26001 study. J Clin Oncol 2011; 29: 134-141

[70] Kocher M, Wittig A, Piroth MD et al. Stereotactic radiosurgery for treatment of brain metastases. A report of the DEGRO Working Group on Stereotactic Radiotherapy. Strahlenther Onkol 2014; 190: 521-532

[71] Yamamoto M, Kawabe T, Sato Y et al. Stereotactic radiosurgery for patients with multiple brain metastases: A case-matched study comparing treatment results for patients with 2-9 versus 10 or more tumors. J Neurosurg 2014; 121 (Suppl): 16-25

[72] Chang WS, Kim HY, Chang JW et al. Analysis of radiosurgical results in patients with brain metastases according to the number of brain lesions: is stereotactic radiosurgery effective for multiple brain metastases? J Neurosurg 2010; 113 (Suppl): 73-78 
[73] Rwigema JC, Wegner RE, Mintz AH et al. Stereotactic radiosurgery to the resection cavity of brain metastases: A retrospective analysis and literature review. Stereotact Funct Neurosurg 2011; 89: 329-337

[74] Specht HMK, Kessel KA, Oechsner M et al. Hypofractionated stereotactic radiotherapy (H-FSRT) to the resection cavity in patients with brain metastases. Strahlenther Onkol 2016; 192: 368-376

[75] Auperin A, Arriagada R, Pignon JP et al. Prophylactic cranial irradiation for patients with small-cell lung cancer in complete remission. Prophylactic Cranial Irradiation Overview Collaborative Group. N Engl J Med 1999; 341: 476-484

[76] Slotman B, Faivre-Finn C, Kramer G et al. Prophylactic cranial irradiation in extensive small-cell lung cancer. N Engl J Med 2007; 357: 664-672

[77] Eriksson PS. Neurogenesis and its implications for regeneration in the adult brain. J Rehabil Med 2003; 17-19
[78] Gondi V, Tome WA, Mehta MP. Why avoid the hippocampus? A comprehensive review. Radiother Oncol 2010; 97: 370-376

[79] Gondi V, Hermann BP, Mehta MP et al. Hippocampal dosimetry predicts neurocognitive function impairment after fractionated stereotactic radiotherapy for benign or low-grade adult brain tumors. Int J Radiat Oncol Biol Phys 2013; 85: 348-354

[80] Gondi V, Tolakanahalli R, Mehta MP et al. Hippocampal-sparing whole-brain radiotherapy: A "how-to" technique using helical tomotherapy and linear accelerator-based intensity-modulated radiotherapy. Int J Radiat Oncol Biol Phys 2010; 78: 1244-1252

[81] Harth S, Abo-Madyan Y, Zheng L et al. Estimation of intracranial failure risk following hippocampal-sparing whole brain radiotherapy. Radiother Oncol 2013; 109: 152-158 